\title{
AV Fistula Thrombosis
}

National Cancer Institute

\section{Source}

National Cancer Institute. AV Fistula Thrombosis. NCI Thesaurus. Code C114756.

Partial or complete occlusion of the lumen within an arteriovenous fistula by a thrombus. 